International Journal of Molecular Medicine and Advance Sciences 6 (3): 34-37, 2010

ISSN: $1813-176 \mathrm{X}$

(C) Medwell Journals, 2010

\title{
Zinc-Related Magnetic Isotope Effect in the Enzymatic ATP Synthesis: A Medicinal Potential of the Nuclear Spin Selectivity Phenomena
}

\author{
${ }^{1}$ Anatoly L. Buchachenko, ${ }^{2}$ Vladimir P. Chekhonin, ${ }^{2}$ Alexey P. Orlov and ${ }^{2}$ Dmitry A. Kuznetsov \\ ${ }^{1}$ N.N. Semenov Institute for Chemical Physics, Russian Academy of Sciences, \\ 4 Kosygin Street, 119991 Moscow, Russian Federation \\ ${ }^{2}$ Department of Medicinal Nanobiotechnologies, N.I. Pirogov Russian State Medical University, \\ 1 Ostrovityanov Street, 117997 Moscow, Russian Federation
}

\begin{abstract}
The rate of enzymatic ATP synthesis is shown to depend on the zinc isotopes. The ATP producing activities of creatine kinase and pyruvate kinase in which $\mathrm{Zn}^{2+}$ ions have magnetic nuclei ${ }^{67} \mathrm{Zn}$ are found to be 2-6 times higher than that of enzymes in which $\mathrm{Zn}^{2+}$ ions have nonmagnetic nuclei ${ }^{64} \mathrm{Zn}$. The isolated rat heart muscle mitochondria exhibit a similar effect. An expression of this magnetic isotope effect in enzymatic ATP synthesis processed in the presence of $\mathrm{Zn}^{2+}$ ions is in a favor to the ion-radical mechanism of ATP production occurred within a high zinc concentration range. Both fundamental aspect and a possible pharmacological significance of the phenomenon described are under discussion.
\end{abstract}

Key words: Hyper activation of the ATP synthesis, magnetic isotope effects, mitochondria function control, phenomenon, $\mathrm{Zn}^{+2}$ ion, nuclei

\section{INTRODUCTION}

The rate of enzymatic ATP synthesis, a Mg involving process was recently shown to strongly depend on the magnesium isotopes. Activity of ATP synthase and ATP producing kinases in which $\mathrm{Mg}^{2+}$ ion has magnetic nucleus ${ }^{25} \mathrm{Mg}$ was found to be $2-3$ times higher than that of enzymes in which $\mathrm{Mg}^{2+}$ ion has nonmagnetic nuclei ${ }^{24} \mathrm{Mg}$ or ${ }^{26} \mathrm{Mg}$ (Buchachenko et al., 2005a-c; Buchachenko, 2009).

The effect was shown to be a function of the concentration of $\mathrm{Mg}^{2+}$ ions at low concentration there is no isotope effect, i.e., classical generally accepted nucleophylic mechanism of the ATP synthesis dominates. If concentration of $\mathrm{Mg}^{2+}$ ions exceeds intracellular one by a few tens a huge isotope effect appears which gives evidence that the new, spin-dependent ion-radical mechanism of ATP synthesis is switched on (Buchachenko et al., 2008). Providing additional and considerable enzymatic source of ATP.

Similar effect was also observed for the calcium ions: the activity of creatine kinase with catalytic sites, loaded with ${ }^{43} \mathrm{Ca}^{2+}$ ions having magnetic nuclei ${ }^{43} \mathrm{Ca}$ was found to be $2.0 \pm 0.3$ times higher than that of enzyme in which $\mathrm{Ca}^{2+}$ ions have even, nonmagnetic nuclei ${ }^{40} \mathrm{Ca},{ }^{42} \mathrm{Ca}$ or ${ }^{44} \mathrm{Ca}$. (Kuznetsov et al., 2010).
Since ATP syntheses, catalyzed by magnesium and calcium are very similar in concentration dependences and isotope effects one can suppose that ion-radical mechanism is a universal phenomenon and may be detected for other metals as catalysts ( $\mathrm{Zn}$ for instance).

\section{MATERIALS AND METHODS}

To verify this idea which would be useful to stimulate ATP production and prevent biomedical pathologies related to deficiency of ATP in the living organisms, we prepared two series of samples of Creatine Kinase (CK), Pyruvate Kinase (PK) and mitochondria. In one series the enzymes were loaded with $\mathrm{Zn}^{2+}$ ions of natural isotope composition in the other one the enzymes were loaded with $\mathrm{Zn}^{2+}$ ions strongly (by $78.4 \%$ ) enriched with magnetic isotope ${ }^{67} \mathrm{Zn}$ (nuclear spin $5 / 2$, magnetic moment $+0.8 \mathrm{mB}$ ). Then both series were tested for their enzymatic activities in the identical conditions.

CK, E.C.2.7.3.2 was isolated from the Vipera xanthia lyophilized venom and purified according to (Kuznetsov et al., 2004). Rabbit reticulocyte PK, E.C.2.6.9.17 was purchased in the ammonium sulfate precipitated form from Worthington, Inc., Durham. The substrates, $\left[{ }^{32} \mathrm{P}\right]$ phosphocreatine, $26.6-29.2 \mathrm{Ci} \mathrm{mmoL}{ }^{-1}$ and $\left[{ }^{32} \mathrm{P}\right]$ phosphoenolpyruvate, $33.6-37.4 \mathrm{Ci} \mathrm{mmoL}^{-1}$ were

Corresponding Author: D.A. Kuznetsov, Department of Medicinal Nanobiotechnologies,

N.I. Pirogov Russian State Medical University, 1 Ostrovityanov Street, 117997 Moscow,

Russian Federation 
manufactured by the Amersham Radiochemical Centre, UK. The enzyme activity A was conventionally evaluated as the amounts of radioactive ${ }^{32} \mathrm{P}$ decays per minute found in the HPLC-separated nascent $\left[{ }^{32} \mathrm{P}\right]$ ATP pool produced by $1.0 \mathrm{mg}$ of pure enzyme during the $40 \mathrm{~min}$ incubation time in the $\mathrm{Mg}^{2+} / \mathrm{Ca}^{2+}$ free samples. This time was shown to be enough for the ATP yield to reach a limiting value. For controls, the metal-free samples incubation at $+37^{\circ} \mathrm{C}$ as well as ice-cold incubation tests were carried out (Buchachenko et al., 2005a; Kuznetsov et al., 1986) the yield of ATP in these experiments was shown to be small in comparison with that in the presence of $\mathrm{Zn}^{2+}$ ions.

The rat heart muscle mitochondria were isolated according to Randall with their following $60 \mathrm{~min}$ long incubation as described by Buchachenko et al. (2005b) to conduct then the oxygen consumption measurements (Rezayat et al., 2009) and the total ATP yield estimations (Buchachenko et al., 2005c). The optimum-balanced mitochondria incubation mixtures (Buchachenko et al., 2005a, b; Rezayat et al., 2009) were employed as they are as well as in their metal-lacking and Zn-supplemented modified forms, same like in experiments with pure enzyme specified above. For protein and DNA quantitative estimations, conventional colorimetric procedures were applied (Bradford, 1976).

Two samples of $\mathrm{ZnCl}_{2}$ were prepared using a routine acidic treatment of the two sorts of zinc oxides: $\mathrm{ZnO}$ with natural isotope composition $\left({ }^{64} \mathrm{Zn}, 48.6 \%\right.$; ${ }^{66} \mathrm{Zn}, 27.9 \%$; ${ }^{67} \mathrm{Zn}, 4.1 \%$; ${ }^{68} \mathrm{Zn}, 18.8 \% ;{ }^{70} \mathrm{Zn}, 0.6 \%$ ) and ${ }^{67} \mathrm{ZnO}$ enriched with magnetic nuclei $\left(78.4 \%\right.$ of ${ }^{67} \mathrm{Zn}$ versus $4.1 \%$ of that in natural $\mathrm{ZnO}$ ), respectively. Enzymatic activity of the free metal CK, PK and mitochondria (the contents of $\mathrm{Ca}^{2+}$ and $\mathrm{Mg}^{2+}$ in the latter were negligibly small, 30-35 and 16-18 $\mu \mathrm{g} \mathrm{mg}^{-1}$ of DNA, respectively) was measured as a function of the $\mathrm{ZnCl}_{2}$ concentration.

\section{RESULTS AND DISCUSSION}

The yield of ATP produced by CK as a function of $\mathrm{ZnCl}_{2}$ concentration is shown in Fig. 1. It exhibits the following remarkable features:

- $\mathrm{Zn}^{2+}$ ions actually catalyze ATP synthesis with the efficiency comparable with that of $\mathrm{Mg}^{2+}$ ions

- ATP yield increases as concentration of $\mathrm{ZnCl}_{2}$ increases then reaches maximum and gradually decreases

- There exists enormously large isotope effect in the ATP synthesis by CK differing in isotope composition

- At high concentration of $\mathrm{ZnCl}_{2}$ both ATP yield and isotope effect are almost completely suppressed

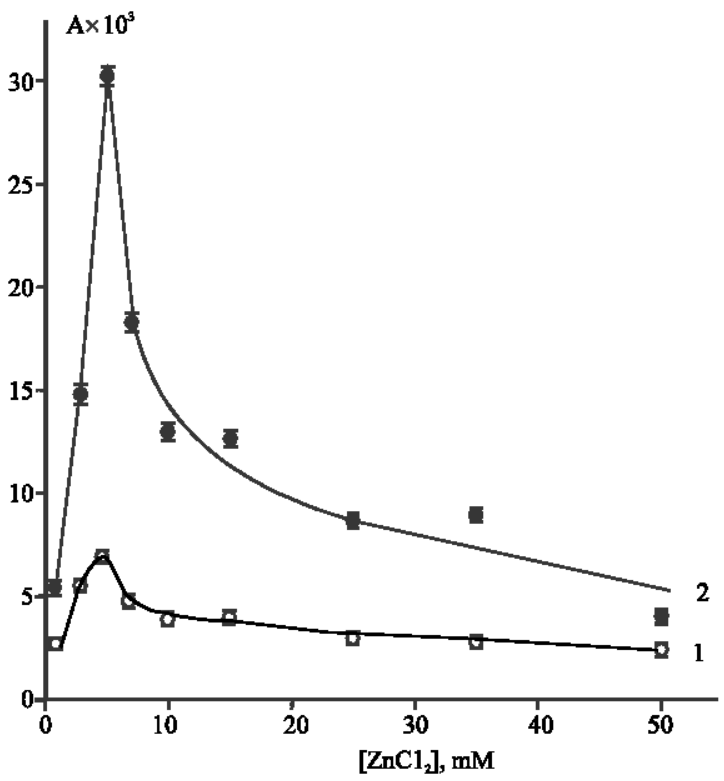

Fig. 1: The yield of ATP produced by $\mathrm{CK}$ as a function of ${ }^{64} \mathrm{ZnCl}_{2}$ (1) and ${ }^{67} \mathrm{ZnCl}_{2}$ (2) concentration

Both samples of $\mathrm{CK}$ contain zinc isotopes in different shares. In the samples with natural $\mathrm{ZnCl}_{2}$ the share of magnetic nuclei ${ }^{67} \mathrm{Zn}$ is $4.1 \%$; the total share of nonmagnetic isotopes is $95.9 \%$ (further we will conventionally denote the set of nonmagnetic isotopes as ${ }^{64} \mathrm{Zn}$ ). In the samples loaded with enriched ${ }^{67} \mathrm{ZnCl}_{2}$ the shares of ${ }^{67} \mathrm{Zn}$ and ${ }^{64} \mathrm{Zn}$ are 78.4 and $21.6 \%$, respectively. Now the activity A of the both samples of CK may be presented as a sum of additive contributions coming from catalytic sites carrying ${ }^{67} \mathrm{Zn}$ and ${ }^{64} \mathrm{Zn}$ :

$$
\begin{aligned}
& \mathrm{A}_{1}=0.041 \mathrm{~A}\left({ }^{67} \mathrm{Zn}\right)+0.959 \mathrm{~A}\left({ }^{64} \mathrm{Zn}\right) \\
& \mathrm{A}_{2}=0.784 \mathrm{~A}\left({ }^{67} \mathrm{Zn}\right)+0.216 \mathrm{~A}\left({ }^{64} \mathrm{Zn}\right)
\end{aligned}
$$

Here $A\left({ }^{67} \mathrm{Zn}\right)$ and $\mathrm{A}\left({ }^{64} \mathrm{Zn}\right)$ characterize the true values of enzymatic activity of catalytic sites with ${ }^{67} \mathrm{Zn}^{2+}$ and ${ }^{64} \mathrm{Zn}^{2+}$ ions, respectively. Substituting into the (Eq. 1, 2) $\mathrm{A}_{1}=7000$ (Fig. 1, curve 1) and $\mathrm{A}_{2}=30200$ (Fig. 1, curve 2) for the $\mathrm{ZnCl}_{2}$ concentration $5 \mathrm{mM}$, it is easy to derive $\mathrm{A}\left({ }^{67} \mathrm{Zn}\right) 37000$ and $\mathrm{A}\left({ }^{64} \mathrm{Zn}\right)=5753$. Their ratio $\mathrm{A}$ $\left({ }^{67} \mathrm{Zn}\right) / \mathrm{A}\left({ }^{64} \mathrm{Zn}\right)=6.4 \pm 0.3$ is the magnitude of isotope effect in enzymatic ATP synthesis by CK. It demonstrates that the CK catalytic sites with ${ }^{67} \mathrm{Zn}^{2+}$ ions produce ATP by 6 times more efficiently than those with ${ }^{64} \mathrm{Zn}^{2+}$ ions.

In order to compare both nuclear spin dependences of the ATP synthesis directed by CK from Vipera xanthia venom and that of the mitochondrial CK promoted one, some additional experiments were carried out using the 


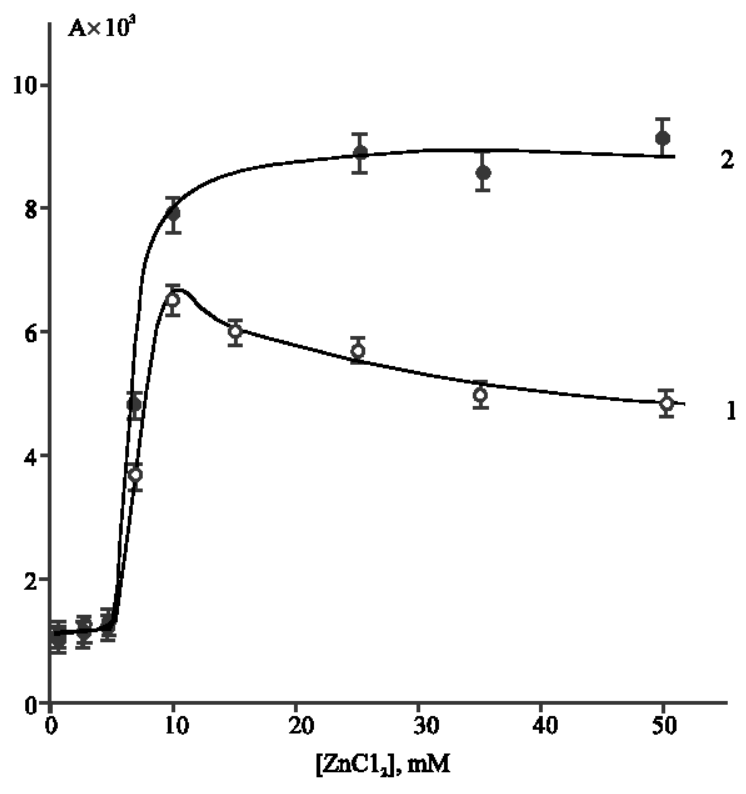

Fig. 2: The yield of ATP produced by $\mathrm{PK}$ as a function of ${ }^{64} \mathrm{ZnCl}_{2}$ (1) and ${ }^{67} \mathrm{ZnCl}_{2}$ (2) concentration

above specified identical standard conditions. As a result, no marked difference found whatsoever. Both CK species tested promotes the very same isotope-related specific activity response whatever metal concentration range studied $(0.5-50.0 \mathrm{mM})$. There is no magnetic isotope effect revealed in the isolated mitochondria oxygen consumption at all it means that isotope effect arises in substrate ATP synthesis itself and has no relation to the NAD-engaging oxidative mitochondrial processes.

ATP synthesis by PK exhibits generally similar but not identical behavior (Fig. 2). The differences are quite evident (Fig. 1 and 2) they assumed to refer to those in molecular mechanics of these two enzymes, $\mathrm{CK}$ and PK. At the concentration of $\mathrm{ZnCl}_{2} 5 \mathrm{mM}$ there is no isotope effect for PK (unlike of CK for which it is 6.4 \pm 0.3 ) however at $50 \mathrm{mM}$ of $\mathrm{ZnCl}_{2}$ it reaches $2.2 \pm 0.3$ (calculated according to Eq. 1 and 2).

Observation of magnetic isotope effect in ATP synthesis catalyzed by $\mathrm{Zn}^{2+}$ ions evidently demonstrates that the ion-radical mechanism of enzymatic ATP synthesis is a universal phenomenon. It includes electron transfer from $\mathrm{Zn}(\mathrm{ADP})^{3 \cdot}$ complex to the hydrated $\mathrm{Zn}$ $\left(\mathrm{H}_{2} \mathrm{O}\right)^{2+}{ }_{n}$ complex as a primary reaction of ATP synthesis. The reaction generates ion-radical pair composed of $\mathrm{Zn}$ $\left(\mathrm{H}_{2} \mathrm{O}\right)_{\mathrm{n}}^{+}$ion and ADP anion-radical coordinated to $\mathrm{Zn}^{2+}$ ion. The addition of the anion-radical to the substrate $\mathrm{P}=\mathrm{O}$ bond results in ATP formation. Populations of the singlet and triplet states and singlet-triplet spin conversion in the pair are controlled by hyperfine coupling of unpaired electrons with magnetic ${ }^{67} \mathrm{Zn}$ and ${ }^{31} \mathrm{P}$ nuclei. Due to this interaction the yield of ATP is a function of nuclear magnetic moment as discussed in detail for magnesium induced ATP synthesis (Buchachenko et al., 2010). For the same reason ATP yield depends on the magnetic field (Buchachenko and Kuznetsov, 2008).

Two results need to be commented. First, the three enzymes, CK from the Vipera xanthia venom, mitochondrial CK and PK exhibit specific isotope effects. Both CK demonstrate ion-radical mechanism of ATP synthesis in the same range of $\mathrm{ZnCl}_{2}$ concentration, however, isotope effects for these two enzymes are slightly different. These differences may be attributed to the differences in molecular structure and dynamics of protein domains in catalytic sites.

Second, large isotope effects are not unexpected and suspicious because they are induced by spin dynamics rather than chemical reactions themselves. Their magnitudes are controlled by the rates of singlet-triplet spin conversion in the ion-radical pairs and depend on the electron-nuclear (hyperfine) coupling of unpaired electrons with magnetic nuclei. In principle, there is no limit on the magnitude of magnetic isotope effect, (Buchachenko, 2009) in contrast to classical, massdependent one which is known to be limited on the ratio of nuclear masses.

\section{CONCLUSION}

Apart from its obvious fundamental significance, the phenomenon described possesses some clear pharmacological potential. Thus, the low toxic cation exchanging nanoparticles formed on a basis of the fullerene-C60 porphyrinic adducts were found to be the reliable carriers for magnetic bivalent metal isotopes suitable for a targeted delivery of the latter's ions in vivo followed then by a marked local hyper-activation of the pre-suppressed ATP synthesis (Rezayat et al., 2009; Amirshahi et al., 2008a).

This alone made a remarkable contribution to either prevention or treatment of several hypoxia related syndromes including the ones associated with some myocardial and lymphoid tissue energy metabolism disorders (Buchachenko, 2009; Kuznetsov et al., 2010; Amirshahi et al., 2008b). As per the phenomena described in a present study, they are no doubt worthy the further nanopharmacological testing in a way offered and pre-programmed recently (Buchachenko, 2009; Kuznetsov et al., 2010; Rezayat et al., 2009; Amirshahi et al., 2008a, b). 


\section{ACKNOWLEDGEMENTS}

This research was financially supported by Russian Ministry of Science and Education (Grant NS-2010.3) and Russian Foundation for Basic Research (Grant 08-0300141). The European INTAS-06/09 Magnetic Field Biological Effects Research Grant is also acknowledged.

\section{REFERENCES}

Amirshahi, N., R.N. Alyautdin, S. Sarkar, S.M. Rezayat and M.A. Orlova et al., 2008b. Fullerene-based low toxic nanocationite particles (porphyrin adducts of cyclohexyl fullerene-C(60)) to treat hypoxia-induced mitochondrial dysfunction in mammalian heart muscle. Arch. Med. Res., 39: 549-556.

Amirshahi, N., R.N. Alyautdin, S. Sarkar, S.M. Rezayat and M.A. Orlova et al., 2008a. New porphyrin adduct of fullerene-C60: A promising nanotool for medicinal use in the heart muscle hypoxia cases. Int. J. Nanosci., 7: 113-135.

Bradford, M.M., 1976. A rapid and sensitive method for the quantitation of microgram quantities of protein utilizing the principle of protein-dye binding. Anal. Biochem., 72: 248-254.

Buchachenko, A.L. and D.A. Kuznetsov, 2008. Magnetic field affects enzymatic ATP synthesis. J. Am. Chem. Soc., 130: 12868-12869.

Buchachenko, A.L., 2009. Magnetic Isotope Effect in Chemistry and Biochemistry. Nova Science Publishers, New York.

Buchachenko, A.L., D.A. Kouznetsov, S.E. Arkhangelsky, M.A. Orlova and A.A. Markarian, 2005a. Spin biochemistry: Intramitochondrial nucleotide phosphorylation is a magnesium nuclear spin controlled process. Mitochondrion, 5: 67-69.

Buchachenko, A.L., D.A. Kouznetsov, S.E. Arkhangelsky, M.A. Orlova and A.A. Markaryan, 2005b. Spin biochemistry: Magnetic $24 \mathrm{Mg}-25 \mathrm{Mg}-26 \mathrm{Mg}$ isotope effect in mitochondrial ADP phosphorylation. Cell Biochem. Biophys., 43: 243-252.
Buchachenko, A.L., D.A. Kouznetsov, M.A. Orlova and A.A. Markarian, 2005c. Magnetic isotope effect of magnesium in phosphoglycerate kinase phosphorylation. Proc. Natl. Acad. Sci. USA., 102: 10793-10796.

Buchachenko, A.L., D.A. Kuznetsov and N.N. Breslavskaya, 2010. Ion-radical mechanism of enzymatic ATP synthesis: DFT calculations and experimental control. J. Phys. Chem. B., 114: 2287-2292.

Buchachenko, A.L., D.A. Kuznetsov, N.N. Breslavskaya and M.A. Orlova, 2008. Magnesium isotope effects in enzymatic phosphorylation. J. Phys. Chem. B., 112: 2548-2556.

Kuznetsov, D.A., A.L. Buchachenko and S.E. Arkhangelsky, 2010. Magnetic isotope effects in the metalloenzymes function control. Proceedings of the 4th SCO Congress on Applied Biochemistry and Biophysics (ABB'10), Ekatherinbourg, Ural Science Press, pp: 202-211.

Kuznetsov, D.A., N.V. Zavijalov, A.V. Govorkov and A.A. Ivanov-Snaryad, 1986. Methyl mercury-induced combined inhibition of ATP regeneration and protein synthesis in reticulocyte lysate cell-free translation system. Toxicol. Lettr., 30: 267-271.

Kuznetsov, D.A., S.E. Arkhangelsky, A.G. Berdieva, P.Z. Khasigov and M.A. Orlova, 2004. A novel electrophoretic technique designed to modify the ratio of magnesium isotopes inside the creatine kinase active sites. A preliminary report. Isotopes Environ. Health Stud., 44: 221-227.

Rezayat, S.M., S.V.S. Boushehri, B. Salmanian, A.H. Omidvari and S. Tarighat et al., 2009. The porphyrin-fullerene nanoparticles to promote the ATP overproduction in myocardium: $25 \mathrm{Mg} 2+-$ magnetic isotope effect. Eur. J. Med. Chem., 44: 1554-1569. 